\title{
Application of Mini-abdominoplasty after Conservative Excision of Extensive Cesarean Scar Endometriosis
}

\author{
Eui Tai Lee ${ }^{1}$, Hyun Min Park ${ }^{1}$, Dong Geun Lee ${ }^{1}$, Kyung Jin Shin ${ }^{1}$, Hak Soon Kim², \\ Ro Hyun Sung ${ }^{3}$, Dong Hee Ryu ${ }^{4}$ \\ Departments of ${ }^{1}$ Plastic and Reconstructive Surgery, ${ }^{2}$ Obstetrics and Gynecology, ${ }^{3}$ Pathology, and ${ }^{4}$ Surgery, Chungbuk National University \\ College of Medicine, Cheongju, Korea
}

Endometriosis is defined as the presence of functioning endometrial tissue outside the uterine cavity, and the standard treatment is extensive surgical excision. Cesarean scar endometriosis is a type of cutaneous endometriosis arising on or near a Cesarean section scar. A 44-year-old woman presented with a $9 \times 6 \mathrm{~cm}$ sized dark-brown, stony-hard, irregular, lower abdominal mass of four years duration. The patient had a history of two Cesarean deliveries, 14 and 16 years ago. Suspecting endometriosis, we excised the tumor conservatively rather than extensively to prevent incisional hernia considering the benign nature of the tumor and the low possibility of recurrence because the patient's age was near menopause, along with simultaneous bilateral salpingo-oophorectomy that was performed in this case. On reconstruction, mini-abdominoplasty was adopted to avoid possible wound complications and cosmetic deformities. The patient was satisfied with the cosmetic results, and neither recurrence nor functional problems occurred during the 1-year follow-up period. Plastic surgeons should keep in mind the possibility of cutaneous endometriosis in an abdominal mass of a female of reproductive age with a previous history of pelvic or intra-abdominal surgery. An optimal result from oncological, functional, and cosmetic standpoints can be achieved with conservative excision followed by mini-abdominoplasty of extensive Cesarean scar endometriosis.

Keywords Endometriosis / Cesarean section / Abdominal wound closure techniques
Correspondence: Eui Tai Lee Department of Plastic and Reconstructive Surgery, Chungbuk National University College of Medicine, 41 Seongbong-ro, Heungdeok-gu, Cheongju 361-711, Korea

Tel: +82-43-269-6095

Fax: +82-43-269-6387

E-mail: etlee@chungbuk.ac.kr

Received: 14 May 2012 • Revised: 20 Jul 2012• Accepted: 2 Aug 2012

pISSN: 2234-6163 • elSSN: 2234-6171 • http://dx.doi.org/10.5999/aps.2012.39.5.551 • Arch Plast Surg 2012;39:551-555

\section{INTRODUCTION}

Endometriosis is a common benign gynecologic disease during the reproductive age due to ectopic proliferation of endometrial tissue under the influence of female hormones outside the uterine cavity. It has a variety of symptoms such as menstrual pain, pelvic pain, dyspareunia, and infertility. Usually endometriosis develops in the uterine adnexa, but sometimes it occurs in an extrapelvic location such as the intestine, lung, liver, pleura, and skin. Cutaneous involvement is less than $1 \%$ of all cases of endometriosis [1-3], and in most cases of such involvement it is found on obstetric and gynecologic surgical sites of the abdomen or perineum following hysterectomy, hysterotomy, Cesarean section, perineotomy, or laparoscopy [4].
This work was supported by a research grant from Chungbuk National

No potential conflict of interest relevant to this article was reported. 
Many cases have been reported in obstetrics, gynecology, and dermatologic journals, but only 1 case has been reported in the plastic and reconstructive surgery field in Korea [5]. The authors experienced an extensive case of endometriosis on a Cesarean section scar, which warranted performing mini-abdominoplasty during reconstruction after conservative excision, saving the abdominal musculoaponeurotic layer in ablation.

\section{CASE}

A 44-year-old woman presented with a slowly growing, goose egg-sized mass on the lower left abdomen and spontaneous pain around the mass associated with profuse menstruation, which first appeared 4 years before her visit. She had undergone a Cesarean delivery twice: 16 years and 14 years earlier. On physical examination, a $9 \times 6 \mathrm{~cm}$ sized dark-brown, lower abdominal mass was observed at the left lateral end of the Cesarean section scar. It was non-tender, irregular, stony-hard, and fixed to both the skin and deep tissue, and thus mimicked a malignant soft tissue tumor of abdominal wall origin (Fig. 1).

On computed tomography, a $68 \times 70 \times 46 \mathrm{~mm}$ heterogeneous enhancing mixed solid and cystic mass was observed at the subcutaneous fat layer of the left lower abdominal wall. It adhered to both the skin and deep fascia, and could not be demarcated from them. Focal penetration of the deep fascia and invasion of the left rectus abdominis and external oblique muscle were seen, leading us to suspect endometriosis or a desmoid tumor. At the same time, a $7 \mathrm{~cm}$ well-defined heterogenous cystic mass was found on the right uterine adnexa tissue leading us to suspect accompanying intrapelvic endometrioma (Fig. 2).

Under general anesthesia, skin incision and dissection around the mass was followed by marginal excisional biopsy. A small amount of endometrial tissue penetrating into the rectus abdominis and external oblique muscle and their fascia was destroyed using electrocoagulation.

The authors performed sharp dissection with a scalpel just above the fascial layer to release the strong adhesion between the mass and deep tissue while preserving the abdominal musculoaponeurotic layer. Incidental rupture of the mass occurred during dissection, but the chocolate-colored material that spilled from the mass was removed with suction and irrigated with distilled water. The excised mass was $8 \times 7.5 \times 6 \mathrm{~cm}$ in size and filled with dark-brown mucous fluid, which appeared to be old liquefied hematoma. Endometriosis was diagnosed based on the presence of endometrial glands and stroma on histopathologic examination (Fig. 3).

The obstetrics and gynecology surgeon then performed total abdominal hysterectomy and bilateral salpingo-oophorectomy.

\section{Fig. 1. Preoperative view}

A 44-year-old woman presented with a $9 \times 6 \mathrm{~cm}$ dark-brown lower abdominal mass that had first appeared 4 years earlier. It was nontender, irregular, stony-hard, and fixed to both skin and deep tissue, and thus mimicked a malignant soft tissue tumor of abdominal wall origin. She had undergone two Cesarean deliveries, 16 years and 14 years before the visit, respectively. (A) Anterior view. (B) Lateral view. (C) Supine position view.
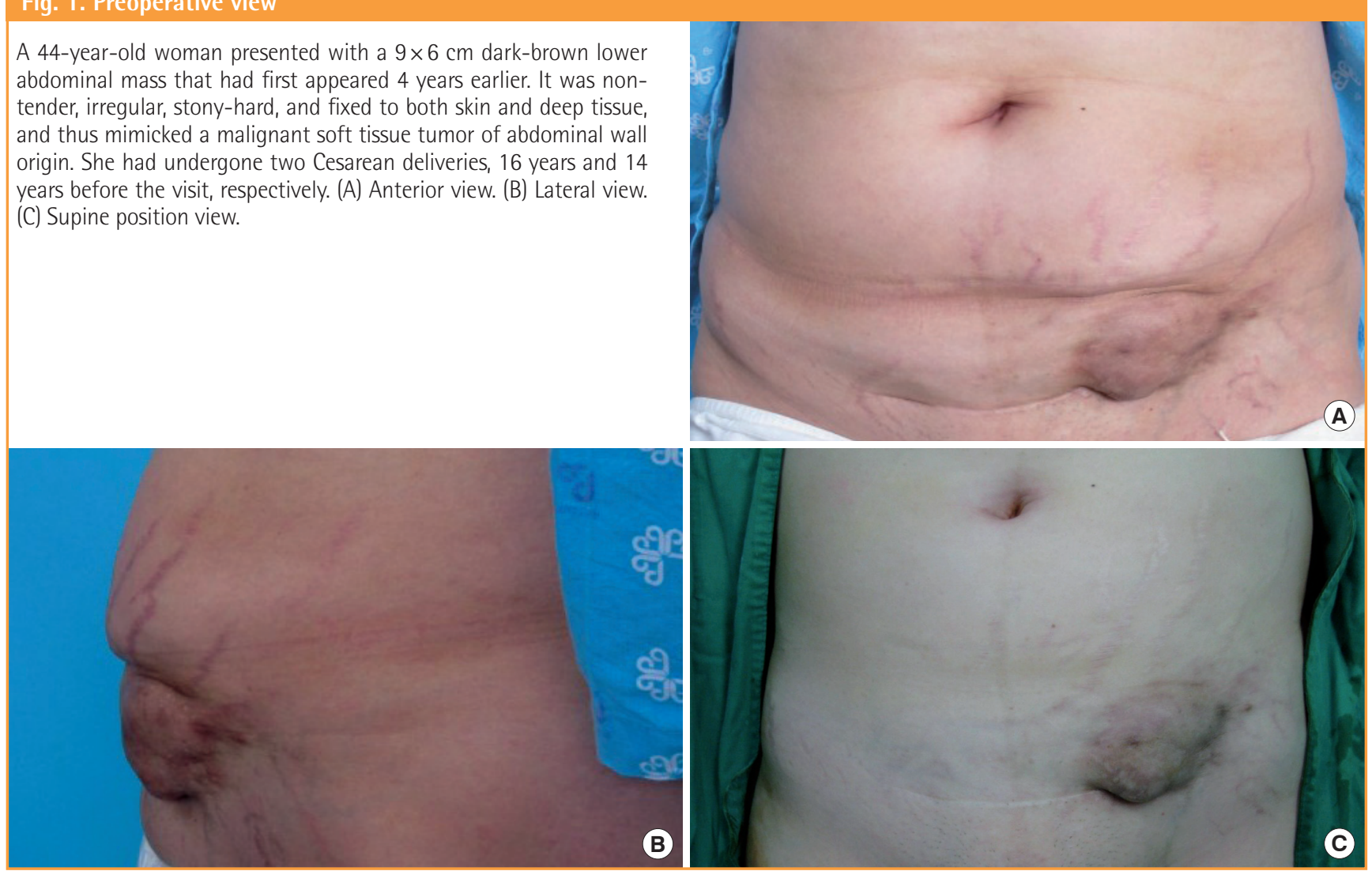
With the musculoaponeurotic buttress saved, mini-abdominoplasty could be applied instead of the author's initial reconstruction plan that consisted of prosthetic mesh reinforcement of the abdominal wall and regional rectus abdominis muscle flap or free flap coverage of the prosthesis. After layer-by-layer repair of the abdominal wall up to the musculoaponeurotic layer, a skinsubcutaneous fat flap was elevated below the umbilicus, and the excessive portion weighted on the right side was trimmed to correct the asymmetry. We repaired the partially damaged rectus abdominis anterior fascia instead of plication of diastasis. Skin closure was completed with progressive tension sutures and a closed suction drain to reduce the dead space (Fig. 4). There was asymmetry of the lengths between the left and right sides of the

\section{Fig. 2. Preoperative abdominopelvic CT image}

The computed tomography (CT) showed a $68 \times 70 \times 46 \mathrm{~mm}$ heterogeneous enhancing solid and cystic mass at the subcutaneous fat layer of the left abdominal wall, which adhered to both the skin and deep fascia, and could not be demarcated from them. Focal penetration of the deep fascia and invasion of the left rectus abdominis and external oblique muscle was observed. A $7 \mathrm{~cm}$ well-defined heterogeneous cystic mass was observed on the right uterine adnexa tissue (white arrow).

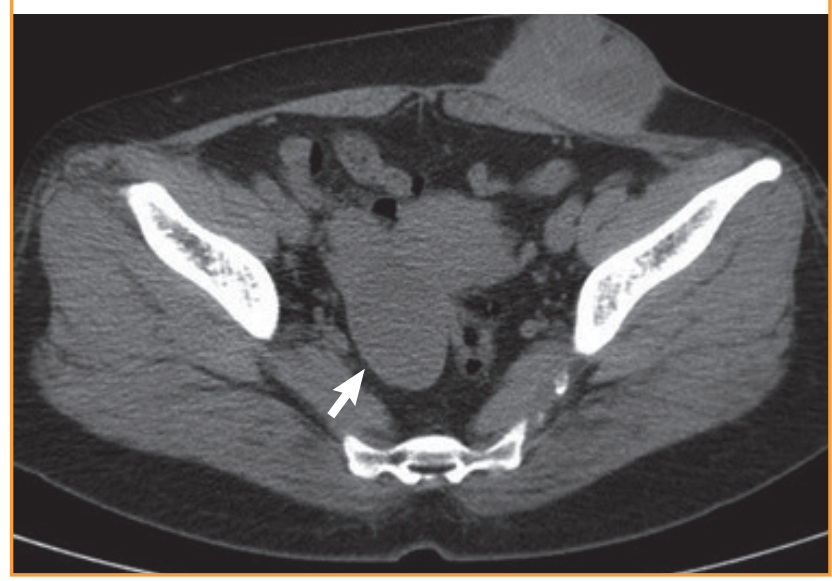

scar, but this was due to the minimized surgical field to ensure a well-formed contour. The patient was satisfied with the cosmetic results, and no evidence of recurrence or clinical problems had occurred by one year after the operation (Fig. 5).

\section{DISCUSSION}

In endometriosis, functioning endometrial gland and stromal tissue is present outside the uterine cavity, and this causes various clinical symptoms because it proliferates under the influence of female hormones [1-3]. The ovary is the most commonly affected site, and the pelvic cavity is the usual location, but the intestine, appendix, gall bladder, pancreas, kidney, ureter, urinary bladder, vagina, perineum, and peritoneum can also be involved [5]. Surgical scar endometriosis involves the incision sites on the abdominal wall, laparoscopic port, perineum, and Bartholin cyst; among them, the incidence of Cesarean scar endome-

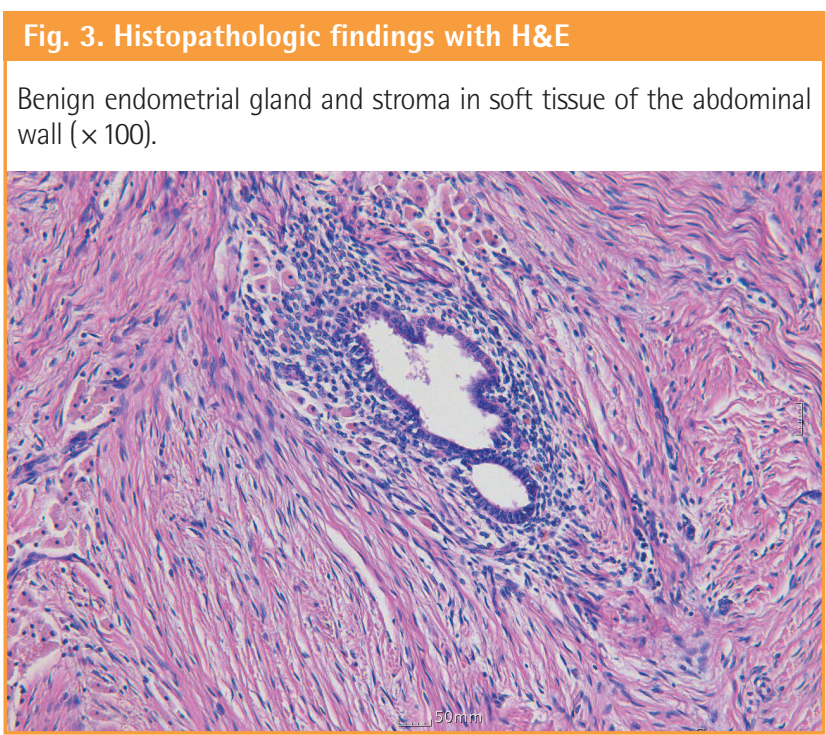

\section{Fig. 4. Intraoperative view}

(A) The mass was excised conservatively including a minimal amount of the rectus abdominis and external oblique fascia. (B) The defect was reconstructed with mini-abdominoplasty.
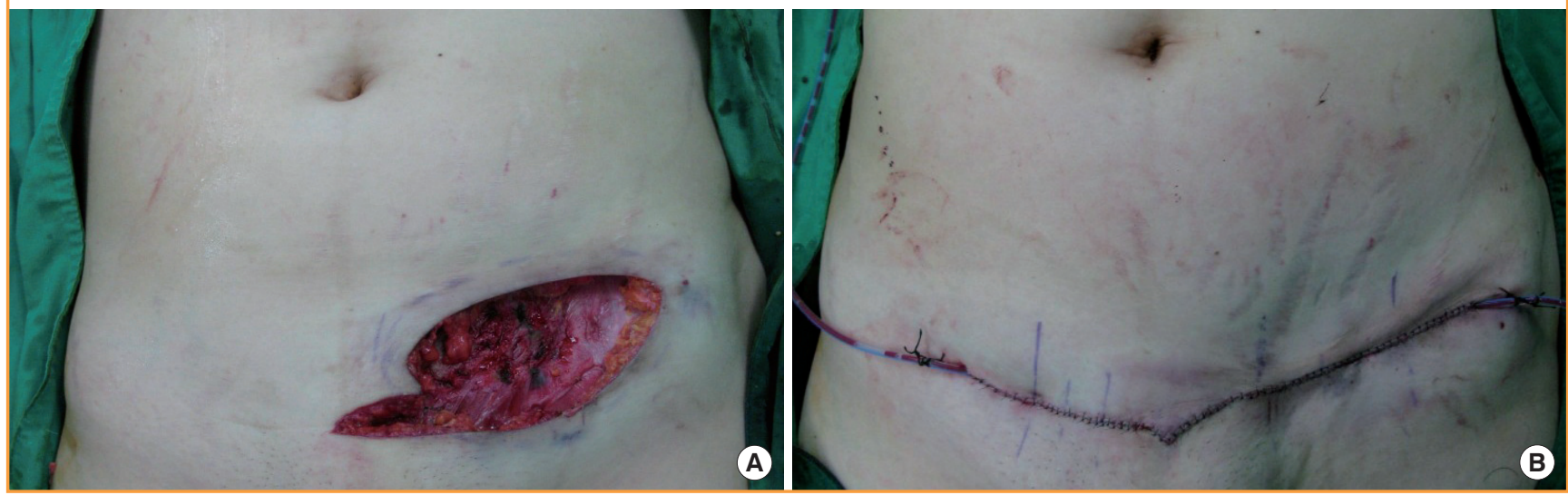


\section{Fig. 5. Postoperative view}

No recurrence or clinical problems were found during the 1-year followup period.

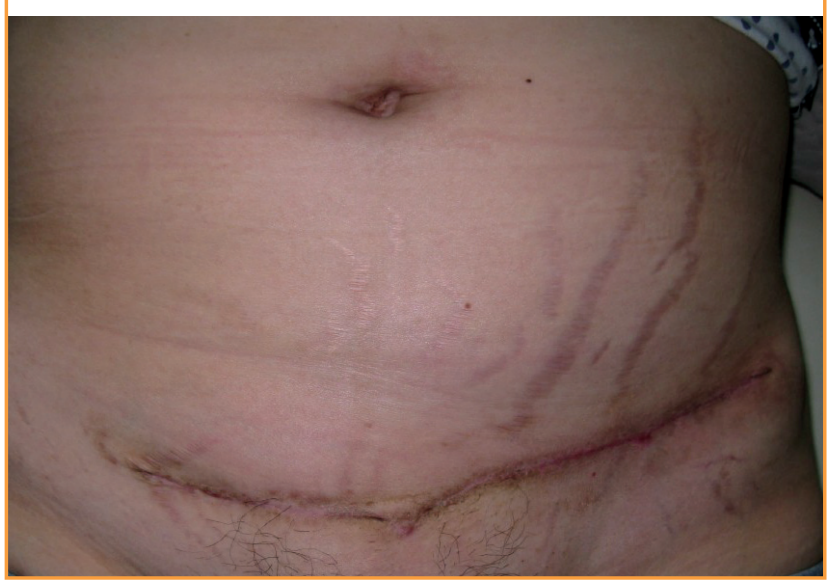

triosis is $0.03 \%$ to $0.4 \%$ [6]. Patients with endometriosis often complain of menstrual pain, dyspareunia, chronic pelvic pain, and infertility. In surgical scar endometriosis, tenderness of the operation site, or a non-tender nodule and periodic pain and bleeding corresponding to the menstrual cycle could be present, as in this case. Differential diagnoses for a mass on a surgical scar are hernia, primary or metastatic tumor, lymphoma, sarcoma, lipoma, abscess, sebaceous cyst, and suture granuloma [2,3]. History taking and physical examination are always the most important procedures for the diagnosis. A nodule on a surgical scar in a reproductive female patient with a prior history of pelvic or intra-abdominal surgery suggests the possibility of surgical scar endometriosis, especially for a lesion with periodic pain on menstruation. The average onset time for surgical scar endometriosis is 30 months after surgery (frequently reported in the literature to be postoperative 1 to 2 years) [7], but it should not be a strict rule because even in this case, ten years had passed before the development of the tumor. Other diagnostic tools including computed tomography, magnetic resonance imaging, fine needle aspiration biopsy, and serum CA-125 could be helpful $[3,5]$. Medication such as a combined oral contraceptive, progesterones, danazol, gonadotropin-releasing hormone agonists, aromatase inhibitors, and androgens can be used for decidualization, atrophy of endometrial tissue, and relieving pain, but recurrence after cessation of medication makes surgical resection the treatment of choice [8-11]. The authors had performed conservative excision on $8 \times 7.5 \times 6 \mathrm{~cm}$ Cesarean scar endometriosis, saving the musculoaponeurotic layer; the defect was reconstructed using mini-abdominoplasty.

The present case highlights four points. First, this is a rare case report of extensive endometriosis. Second, scar endometriosis can be misdiagnosed as a malignant tumor, so plastic surgeons should keep in mind the possibility of cutaneous endometriosis in an abdominal mass of a reproductive female with a previous history of pelvic or intra-abdominal surgery. Third, though wide en bloc excision with a surrounding safety margin is the standard surgical method to prevent local recurrence of endometriosis as like a malignancy, the authors decided to perform conservative excision of the possible remnant tumor instead of wide excision in consideration of the benign nature of the disease and the low probability of recurrence with regard to the patient's age nearing menopause along with the simultaneous bilateral salpingooophorectomy. In our patient, the mass abutted and even penetrated the rectus abdominis, external oblique, and pyramidalis muscles and their fascia due to adhesion at the surgical site and frequent menstrual bleeding. If conventional wide excision were performed at the sacrifice of the musculoaponeurotic strut, an incisional hernia could have occurred in the lower abdominal wall, which lacked the posterior sheath of the rectus abdominis. Finally, we performed mini-abdominoplasty to avoid functional and cosmetic problems. The large defect after ablation of the tumor made the primary closure difficult and could cause various wound complications such as dehiscence, hematoma, seroma, and hypertrophic scar; asymmetry of the abdominal wall or dog-ear deformities could also occur. In mini-abdominoplasty, the dissection is limited to below the umbilicus, and a smaller amount of excessive skin and subcutaneous tissue is resected via a shorter incision [12]. Through limited dissection, many complications associated with full-abdominoplasty such as hematoma, seroma, necrosis of skin flap, and umbilicus and malposition of the umbilicus can be reduced. The application of mini-abdominoplasty made the patient satisfied with eradication of both the tumor and lower abdominal obesity without any of the problems associated with primary closure or full-abdominoplasty.

\section{REFERENCES}

1. Gajjar KB, Mahendru AA, Khaled MA. Caesarean scar endometriosis presenting as an acute abdomen: a case report and review of literature. Arch Gynecol Obstet 2008;277: 167-9.

2. Francica G, Giardiello C, Angelone G, et al. Abdominal wall endometriomas near cesarean delivery scars: sonographic and color doppler findings in a series of 12 patients. J Ultrasound Med 2003;22:1041-7.

3. Wolf GC, Singh KB. Cesarean scar endometriosis: a review. Obstet Gynecol Surv 1989;44:89-95.

4. Kim JK, Cha SI, Lee YS, et al. A case of primary cutaneous endometriosis at the unbilicus. Korean J Obstet Gynecol 2005;48:1064-8. 
5. Yi TM, Kim YJ, Kim SG, et al. A case of endometrioma at the abdominal scar. J Korean Soc Plast Reconstr Surg 2010; 37:843-6.

6. Hong HJ, Chung BS, Son EJ, et al. Abdominal wall endometriosis after Cesarean section: a report of two cases. Korean J Obstet Gynecol 2005;48:228-33.

7. Jeong YJ, Kim JC. Endometrioma in the abdominal incisional scar. J Korean Surg Soc 2003;65:259-62.

8. Kang AR, Hur JY. Medical therapy of endometriosis. J Korean Med Assoc 2010;53:424-9.
9. Elbiss HM, Thomson AJ. The management of stage III and IV endometriosis. Arch Gynecol Obstet 2012;285:387-96.

10. Falcone T, Lebovic DI. Clinical management of endometriosis. Obstet Gynecol 2011;118:691-705.

11. Schindler AE. Hormonal contraceptives and endometriosis/adenomyosis. Gynecol Endocrinol 2010;26:851-4.

12. Sim HB, Yoon SY. Experiences of abdominoplasty without undermining. J Korean Soc Plast Reconstr Surg 2006;33: 303-7. 